\title{
Diagnostic utility of low hemoglobin density to detect iron deficiency in patients with inflammatory bowel disease
}

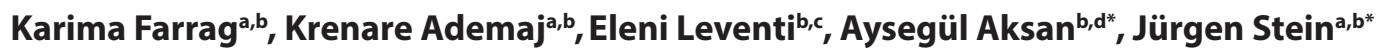 \\ DGD Kliniken Sachsenhausen, Frankfurt am Main; Interdisziplinäres Crohn Colitis Centrum Rhein-Main, Frankfurt \\ am Main; Klinikum Hanau; Institute of Nutritional Science, Justus-Liebig University Giessen, Germany
}

Abstract

Background In the absence of a feasible noninvasive gold standard, iron deficiency (ID) anemia (IDA) is best measured using multiple indicators. However, the choice of an appropriate single iron biomarker for ID screening continues to be debated. Low hemoglobin density (LHD\%) from Coulter counters has been suggested as a useful tool to detect ID. This study investigated the reliability of $\mathrm{LHD} \%$ for the assessment of iron status in patients with inflammatory bowel disease (IBD) and IDA, anemia of chronic disease (ACD) or mixed anemia (MIX).

Methods The study population consisted of 143 patients with IBD (aged $39.03 \pm 12.53$ years, $61.5 \%$ female). Blood count, transferrin saturation, serum ferritin, and C-reactive protein were determined by routine assays. Patients with anemia were divided into 3 groups: IDA, ACD and MIX, according to specific criteria. Receiver operator characteristic (ROC) curves were constructed.

Results ROC analysis for LHD\% in the detection of ID yielded a cutoff value of $3.8 \%$. In anemic patients, LHD\% values did not differ statistically significantly between groups (IDA, ACD, MIX) and no significant difference in LHD\% values was observed between patients with IDA and ID.

Conclusions These results demonstrate that LHD\% is a reliable biomarker for the detection of iron deficiency in patients with IBD and anemia, regardless of whether inflammation is present. Our findings indicate that $\mathrm{LHD} \%$ can provide added value in diagnosing iron deficiency.

Keywords Inflammatory bowel disease, iron deficiency, low hemoglobin density

Ann Gastroenterol 2021; 34 (1): 1-7

${ }^{\mathrm{a}}$ Gastroenterology and Clinical Nutrition, DGD Kliniken Sachsenhausen, Frankfurt am Main (Karima Farrag, Krenare Ademaj, Jürgen Stein); ' Interdisziplinäres Crohn Colitis Centrum Rhein-Main, Frankfurt am Main (Karima Farrag, Krenare Ademaj, Eleni Leventi, Aysegül Aksan, Jürgen Stein); 'Department of Gastroenterology, Klinikum Hanau (Eleni Leventi); ${ }^{\mathrm{d}}$ Institute of Nutritional Science, Justus-Liebig University Giessen (Aysegül Aksan), Germany

Conflicts of Interest: KF: speakers' honoraria Immundiagnostik AG. KA, EL: No conflicts of interest. AA: Research funding, consulting and congress fees from Immundiagnostik AG and Vifor. JS: consultancy fees from Immundiagnostik AG, payment for lectures from Immundiagnostik AG

${ }^{\star}$ Contributed equally to this work

Correspondence to: Karima Farrag, Dept. of Gastroenterology and Clinical Nutrition, DGD Kliniken Sachsenhausen, Schulstrasse 31, Frankfurt am Main, Germany, e-mail: farrag@gmx.de

Received 30 November 2020; accepted 31 December 2020; published online 2 April 2021

DOI: https://doi.org/10.20524/aog.2021.0622

\section{Introduction}

The prevalence of iron deficiency (ID) in patients with inflammatory bowel disease (IBD) has been reported at 36$76 \%$, with approximately $50 \%$ of patients having isolated ID without anemia. Since ID, even without manifest anemia, can substantially impact quality of life and healthcare costs, IBDassociated ID requires appropriate diagnostic and therapeutic management [1-4].

Two different types of impaired iron homeostasis may be differentiated: absolute ID and functional ID. In absolute ID, iron stores are depleted as a result of insufficient dietary iron intake, iron malabsorption and/or chronic gastrointestinal blood loss [5,6]. ID anemia (IDA) occurs when iron stores are fully depleted and iron supply is insufficient for hemoglobin $(\mathrm{Hb})$ synthesis [7]. In functional ID, iron supply is inadequate to ensure sufficient hemoglobinization of reticulocytes and mature erythrocytes. This imbalance between erythroid 
marrow iron requirements and actual iron supply inhibits hemoglobinization, resulting in hypochromic mature red cells and reticulocytes $[8,9]$. Despite a plethora of iron status parameters, screening for ID remains challenging. Serum ferritin (s-ferritin) indirectly estimates body iron stores and is the most specific biomarker of absolute ID. Absolute ID in patients with IBD may, however, be obscured by the acute-phase response induced by inflammation and/or infection, and as a result of anemia of chronic disease (ACD), characterized by apparently normal or increased iron stores but limited iron available for erythropoiesis (iron-restricted erythropoiesis) [10].

The dual "face" of ID in IBD is a conundrum for the IBD physician, as its 2 main subgroups (IDA and ACD) frequently mix and overlap. Conventional iron status parameters, such as mean cell volume, transferrin saturation (TSAT) and s-ferritin [10-12], are of limited diagnostic value in this setting because of dynamic changes (e.g., acute-phase response), and thus fail to accurately reflect changes in the red blood cell population because of the lack of temporal coincidence [13]. Higher cutoff levels for s-ferritin in the presence of infection/ inflammation have been investigated, though these are not evidence-based [14]. In the absence of a feasible noninvasive gold standard, European guidelines recommend the use of multiple indicators to detect IDA [15]. Thus, there is a demand for markers suitable for the early and reliable diagnosis of ID, but uninfluenced by inflammation.

Low $\mathrm{Hb}$ density (LHD\%), derived from the traditional marker mean corpuscular $\mathrm{Hb}$ concentration (MCHC), has been shown to be a highly sensitive biomarker for iron availability during erythropoiesis. MCHC is an allencompassing parameter for the availability of iron during the previous 90-120 days, as well as for the regular use of iron in intracellular Hb. Similarly, LHD\% reflects iron availability and hemoglobinization of mature red blood cells [16-18].

Despite its potential clinical utility, no previous studies have evaluated LHD\% in patients with IBD. We therefore aimed to investigate its diagnostic performance in the detection of ironrestricted erythropoiesis in patients with IBD.

\section{Patients and methods}

This study was conducted as a comparative, cross-sectional, retrospective study according to the ethical principles of the Declaration of Helsinki in adult patients previously diagnosed with IBD and healthy controls. The study design was approved by the local ethics committee, the Landesärztekammer Hessen (2019-1317-evBO). All patients were required to give informed written consent prior to participation.

\section{Study population}

The data from eligible patients from the Interdisciplinary Crohn Colitis Center (ICCC) Rhein-Main (Frankfurt am Main, Germany) who had been diagnosed with IBD according to standard clinical, endoscopic, radiological and pathological criteria were retrospectively analyzed. The control group consisted of healthy adults attending the same center for routine consultations. Inclusion criteria were age $18-65$ years and body mass index $(\mathrm{BMI})<30 \mathrm{~kg} / \mathrm{m}^{2}$. Exclusion criteria were malignant underlying disease, severe metabolic disease, cardiac, pulmonary, renal or hepatic dysfunction or disease, substance abuse (alcohol, drugs), oral or intravenous iron supplementation within 3 months prior to baseline, azathioprine-induced cytopenia, and blood donation, blood loss or plasmapheresis within the last 3 months prior to the laboratory tests. Also excluded were patients for whom laboratory values were missing.

\section{Study design}

Eligible patients and controls were identified retrospectively. Prior to data extraction, the patients were contacted, fully informed about all aspects of the study and, if they were willing to participate, asked to give written informed consent. Data were then drawn from electronic patient records. The documented information included demographics, a full blood count including $\mathrm{Hb}$, s-ferritin, TSAT, erythrocyte sedimentation rate (ESR), and serum high-sensitivity C-reactive protein (hsCRP). All blood samples were collected in the morning, after 12-h fasting, and were analyzed the same day. All laboratory findings were determined by routine assays.

Disease location was defined according to the Montreal Classification [19]. ID was defined as s-ferritin $<30 \mu \mathrm{g} / \mathrm{L}$ and TSAT $<20 \%$. Functional ID was defined as hsCRP $>5 \mathrm{mg} / \mathrm{L}$, s-ferritin between $30-300 \mathrm{mg} / \mathrm{L}$ and TSAT $<20 \%$ [15]. Anemia was defined as an $\mathrm{Hb}$ concentration below $13 \mathrm{~g} / \mathrm{dL}$ for males and $12 \mathrm{~g} / \mathrm{dL}$ for females, according to World Health Organization criteria $[20,21]$, and subclassified by type: as IDA if hsCRP $<5 \mathrm{mg} / \mathrm{L}$, TSAT $<20 \%$ and s-ferritin $<30 \mu \mathrm{g} / \mathrm{L}$; as ACD if hsCRP $\geq 5 \mathrm{mg} / \mathrm{L}$, TSAT $<20 \%$, and s-ferritin $\geq 100 \mu \mathrm{g} / \mathrm{L}$; and as mixed IDA and ACD (MIX) if hsCRP $\geq 5 \mathrm{mg} / \mathrm{L}$, TSAT $<20 \%$ and s-ferritin level $30-100 \mu \mathrm{g} / \mathrm{L}[22,23]$.

LHD\% was calculated from the traditional MCHC using the mathematical sigmoid transformation recently proposed as a new ID parameter by Beckman-Coulter (Beckman Coulter Inc., Miami, FL, USA) [16]:

$$
\mathrm{LHD} \%=100 \sqrt{1-\left[1 /\left(1+\mathrm{e}^{1.8(30-\mathrm{MCHC})}\right)\right]}
$$

\section{Statistical analysis}

Statistical analysis was performed using IBM SPSS version 25.0. LHD\% was calculated using the Excel formula function. All variables were investigated for normal distribution using both visual and analytical methods. Descriptive parameters were presented as median and range. Appropriate parametric or non-parametric tests were performed based on a normal distribution. Receiver operating characteristic (ROC) curve 
analysis was used to evaluate the diagnostic performance of LHD\% for the assessment of iron status. Statistical significance was predetermined as $\mathrm{P}<0.05$.

\section{Results}

The study population consisted of 192 subjects: 143 patients with IBD (78 Crohn's disease, 65 ulcerative colitis, mean age $39.03 \pm 12.53$ years, $88 / 143$ female) and 49 healthy controls (mean age $42.37 \pm 12.23$ years, $34 / 49$ female). Of the patients with IBD, 38 were in a non-inflammatory and nonanemic state (mean age $39.47 \pm 11.86$ years, $24 / 38$ female) based on their hsCRP and Hb levels (Table 1). There was no statistical significance between the groups with regard to sex, age, age at diagnosis, disease location, disease behavior or drug therapies.
Baseline laboratory parameters of the IBD group according to iron status are shown in Table 2. Of the 143 patients with IBD, 105 (73.4\%) were iron deficient; of these, 79 (75.2\%) had absolute and $26(24.8 \%)$ functional ID. Sixty-five of these iron deficient patients $(62.0 \%)$ were anemic, of whom 44 (67.7\%), 8 (12.3\%) and 13 (20\%) fulfilled the predefined criteria for IDA, MIX, and ACD, respectively. In accordance with the selection criteria, median hsCRP and ESR values were significantly higher in the MIX and ACD groups compared to the IDA group, and in the patients with functional ID compared to those with absolute ID.

A ROC analysis was performed for LHD\%, and the optimal cutoff and area under the curve (AUC) for defining ID (absolute and functional) were calculated. Functional and absolute ID are presented in Table 3, together with results of the ROC analysis for ferritin (cutoff: $30 \mathrm{ng} / \mathrm{mL}$ for ID and absolute ID, $100 \mathrm{ng} / \mathrm{mL}$ for functional ID) and TSAT (cutoff: 20\%). The optimal cutoff for LHD\% was

Table 1 Subject characteristics

\begin{tabular}{|c|c|c|c|c|c|c|}
\hline \multirow[t]{2}{*}{ Characteristics } & \multicolumn{3}{|c|}{ Patients with IBD $(\mathrm{n}=143)$} & \multirow{2}{*}{$\begin{array}{l}\text { Control Group } \\
(\mathrm{n}=49)\end{array}$} & \multirow[t]{2}{*}{$\mathrm{P}$-value ${ }^{2}$} & \multirow[t]{2}{*}{ P-value } \\
\hline & $\begin{array}{l}\text { Iron deficient } \\
\text { and/or anemic }\end{array}$ & $\begin{array}{l}\text { Non-inflammatory and } \\
\text { non-iron deficient }\end{array}$ & P-value ${ }^{1}$ & & & \\
\hline $\mathrm{N}(\&)$ & $105(64)$ & $38(24)$ & 0.811 & $49(34)$ & 0.311 & 0.541 \\
\hline Age $($ mean \pm SD) & $39.02 \pm 12.90$ & $39.47 \pm 11.86$ & 0.791 & $42.37 \pm 12.23$ & 0.098 & 0.221 \\
\hline $\begin{array}{l}\text { Disease type } \\
\text { CD } \\
\text { UC }\end{array}$ & $\begin{array}{l}59(56.2 \%) \\
46(43.8 \%)\end{array}$ & $\begin{array}{l}19(50.0 \%) \\
19(50.0 \%)\end{array}$ & 0.511 & & & \\
\hline $\begin{array}{l}\text { Age at diagnosis } \\
\text { A1 }=\text { below } 16 \text { years } \\
\text { A2 }=17 \text { to } 40 \text { years } \\
\text { A3 }=\text { over } 40 \text { years }\end{array}$ & $\begin{array}{c}1(1.0 \%) \\
66(62.9 \%) \\
37(35.2 \%)\end{array}$ & $\begin{array}{c}- \\
21(55.3 \%) \\
17(44.7 \%)\end{array}$ & 0.526 & & & \\
\hline $\begin{array}{l}\text { Disease location } \\
\text { L1 }=\text { Terminal ileum (CD) } \\
\text { L2 }=\text { Colon }(\mathrm{CD}) \\
\text { L3 }=\text { Ileocolon }(\mathrm{CD}) \\
\text { L4 }=\text { Upper gastrointestinal tract (CD) } \\
\text { E1 }=\text { Proctitis (UC) } \\
\text { E2 }=\text { Left-sided colitis (UC) } \\
\text { E3 }=\text { Pancolitis (UC) }\end{array}$ & $\begin{array}{c}21(37.3 \%) \\
17(30.5 \%) \\
16(28.6 \%) \\
2(3.6 \%) \\
4(8.4 \%) \\
28(58.3 \%) \\
16(33.3 \%)\end{array}$ & $\begin{array}{c}9(47.4 \%) \\
5(26.3 \%) \\
5(26.3 \%) \\
- \\
4(21.1 \%) \\
11(57.8 \%) \\
4(21.1 \%)\end{array}$ & $\begin{array}{l}0.770 \\
0.282\end{array}$ & & & \\
\hline $\begin{array}{l}\text { Disease behavior }(\mathrm{CD}) \\
\mathrm{B} 1=\text { non-stricturing, non-penetrating } \\
\mathrm{B} 2=\text { stricturing } \\
\mathrm{B} 3=\text { penetrating } \\
\mathrm{B} 4=\text { perianal }\end{array}$ & $\begin{array}{l}29(51.8 \%) \\
11(19.6 \%) \\
16(28.6 \%) \\
-\end{array}$ & $\begin{array}{c}12(63.2 \%) \\
6(31.6 \%) \\
1(5.2 \%) \\
-\end{array}$ & 0.099 & & & \\
\hline $\begin{array}{l}\text { IBD treatments } \\
\text { 5-ASA } \\
\text { Steroids } \\
\text { Azathioprine } \\
\text { Methotrexate } \\
\text { Anti-TNF } \\
\text { Ustekinumab }\end{array}$ & $\begin{array}{c}17(16.2 \%) \\
32(30.5 \%) \\
18(17.2 \%) \\
6(5.7 \%) \\
38(36.2 \%) \\
11(10.5 \%)\end{array}$ & $\begin{array}{c}13(34.2 \%) \\
7(18.4 \%) \\
5(13.2 \%) \\
0(0.0 \%) \\
14(36.8 \%) \\
3(7.9 \%)\end{array}$ & 0.055 & & & \\
\hline
\end{tabular}

One sample t-test for age, chi-square test for all the other comparisons; P-value ${ }^{1}$ : comparison of iron deficient and/or anemic IBD patients and noninflammatory and non-iron deficient IBD patients; P-value ${ }^{2}$ : comparison of iron deficient and/or anemic IBD patients and control group; P-value ${ }^{3}$ : comparison of non-inflammatory and non-iron deficient IBD patients and control group

IBD, inflammatory bowel disease; CD, Crohn's disease; UC, ulcerative colitis; 5-ASA, 5-aminosalicylic acid; TNF, tumor necrosis factor; SD, standard deviation 
Table 2 Laboratory markers according to iron status in patients with IBD

\begin{tabular}{lcccccc}
\hline Markers & Absolute ID (n=79) & Functional ID (n=26) & P-value $^{1}$ & IDA (n=44) & ACD or MIX (n=21) $^{*}$ P-value \\
\hline Hb (g/dL) & $12.5(7.4-16.2)$ & $11.4(9.5-130)$ & $0.012^{*}$ & $11.0(7.4-13.3)$ & $11.8(9.2-12.9)$ & 0.311 \\
RDW (\%) & $14.6(11.9-22.5)$ & $14.8(11.7-21.2)$ & 0.894 & $15.2(12.5-20.8)$ & $15.0(11.7-21.2)$ & 0.776 \\
MCHC (g/dL) & $32.8(28.6-35.7)$ & $32.3(29.9-34.3)$ & 0.190 & $31.9(28.6-34.0)$ & $32.2(29.9-34.3)$ & 0.424 \\
Ferritin (ng/mL) & $15.1(5.0-29.1)$ & $94.1(30.5-1028.0)$ & $<0.001$ & $13.9(5.0-28.4)$ & $124.0(30.5-1028.0)$ & $<0.001$ \\
TSAT (\%) & $9.5(2.5-36.1)$ & $14.1(2.4-25.6)$ & 0.123 & $5.7(2.5-21.9)$ & $12.2(2.4-25.6)$ & $<0.001$ \\
LHD (\%) & $8.0(0.6-96.2)$ & $13.1(2.1-73.8)$ & 0.184 & $17.8(2.7-96.2)$ & $13.7(2.1-73.8)$ & 0.424 \\
ESR (\%) & $14.0(1.0-79.0)$ & $25.5(2.0-89.0)$ & $<0.001$ & $20.0(2.0-79.0)$ & $26.0(2.0-89.0)$ & $0.018^{*}$ \\
HsCRP (mg/L) & $2.7(1.0-24.4)$ & $13.4(1.4-181.0)$ & $<0.001$ & $2.7(1.0-24.4)$ & $27.5(1.4-181.0)$ & $<0.001$ \\
\hline
\end{tabular}

${ }^{*} \mathrm{P}<0.05,{ }^{*} \mathrm{P}<0.001$, Mann-Whitney U test

P-value ${ }^{1}$ : difference between absolute ID group and functional ID group; P-value ${ }^{2}$ : difference between IDA group and ACD or MIX group

$I B D$, inflammatory bowel disease; Hb, hemoglobin; RDW, red cell distribution width; MCHC, mean corpuscular hemoglobin concentration; TSAT, transferrin saturation; LHD, low hemoglobin density; ESR, erythrocyte sedimentation rate; HsCRP, high-sensitivity C-reactive protein; ID, iron deficiency; IDA, iron deficiency anemia; $A C D$, anemia of chronic disease; MIX, mixed anemia

Table includes only classified anemia - there were patients with unclassified ID ( $\mathrm{n}=1)$ and IDA $(\mathrm{n}=8)$

Table 3 Analytical performance of iron status parameters to detect patients with ID, absolute ID, and functional ID as sole biomarker

\begin{tabular}{lccc}
\hline Iron status & Ferritin & TSAT & LHD \\
\hline ID & & & \\
Sensitivity & $75 \%$ & $78 \%$ & $82 \%$ \\
Specificity & $89 \%$ & $84 \%$ & $85 \%$ \\
AUC ${ }^{\text {ROC }}$ & 0.860 & 0.905 & 0.872 \\
Cutoff & $30 \mathrm{ng} / \mathrm{mL}$ & $20 \%$ & $3.8 \%$ \\
Absolute ID & & & \\
Sensitivity & $100 \%$ & $77 \%$ & $79 \%$ \\
Specificity & $91 \%$ & $69 \%$ & $70 \%$ \\
AUC & 0.989 & 0.830 & 0.754 \\
Cutoff & $30 \mathrm{ng} / \mathrm{mL}$ & $20 \%$ & $3.8 \%$ \\
Functional ID & & & \\
Sensitivity & $50 \%$ & $80 \%$ & $89 \%$ \\
Specificity & $24 \%$ & $55 \%$ & $70 \%$ \\
AUCROC & 0.250 & 0.675 & 0.763 \\
Cutoff & $100 \mathrm{ng} / \mathrm{mL}$ & $20 \%$ & $3.8 \%$ \\
\hline
\end{tabular}

ID, iron deficiency; TSAT, transferrin saturation; LHD, low hemoglobin density; $A U C^{R O C}$ area under curve receiver operating characteristic

identified to be $3.8 \%$, regardless of inflammatory status. This cutoff value was confirmed to be valid in all 3 analyses, with different sensitivity and specificity levels (Table 3). For ID (both absolute and functional), the AUC was 0.872 with $82 \%$ sensitivity and $85 \%$ specificity; for absolute ID, the AUC was 0.754 with $79 \%$ sensitivity and $70 \%$ specificity; and for functional ID, the AUC was 0.763 with $89 \%$ sensitivity and $70 \%$ specificity. Both ferritin and TSAT were poor predictors of functional ID in comparison to LHD\%.

Taking 3.8\% as an optimal cutoff point for LHD\%, as suggested by the ROC analysis (sensitivity $82 \%$, specificity $85 \%$ ) for ID detection, $82.3 \%$ and $88.5 \%$ of patients with absolute ID and functional ID, respectively, had elevated LHD\% values. In the anemic group, $97 \%$ of patients with IDA, $100 \%$ with MIX, and $84.6 \%$ with ACD had elevated levels of LHD\% (Fig. 1).

\section{Discussion}

ID, with or without anemia, is recognized as one of the most common complications and extraintestinal manifestations of IBD. ID, even without anemia, can be debilitating, affecting overall health and daily work and social life.

Laboratory diagnostics of ID are currently based largely on low levels of TSAT and s-ferritin. However, inflammation is well known to mimic some aspects of ID by impairing the utilization of existing iron stores for red cell production and inducing an iron-sequestration syndrome and hypoferremia [24]. Therefore, diagnosing ID in patients affected by chronic inflammatory diseases such as IBD is a major diagnostic challenge. Additionally, chronic inflammation hinders the interpretability of other parameters of low iron stores [13,25]. Inflammationindependent diagnosis of true ID is of clinical importance when choosing the treatment approach and obviates unnecessary treatment-related adverse events that may occur as a result of unnecessary iron supplementation therapy [26].

Hepcidin has been proposed as a possible tool in this context. However, the antibodies used in diagnostic immunoassays can cross-react with biologically inactive hepcidin and thus overestimate true bioactive hepcidin, while there are additional in vivo factors that induce or prevent iron-restricted hemopoiesis with or without the expected hepcidin reaction [27].

In recent years, a variety of other biomarkers have been studied for their utility as indicators of true ID in the context of inflammation. One of these laboratory parameters is the serum concentration of soluble transferrin receptor (sTfR), an indicator of the iron requirement for iron erythropoiesis. However, transferrin receptor expression on cells is also influenced by inflammation, which may negatively affect the sensitivity of sTfR levels as an indicator of true ID in patients with inflammatory disorders $[22,28]$. The calculated ratio, transferrin receptor: $\log _{10}$ ferritin (TfR-F), has been also proposed as a reliable marker and has been shown to offer superior discrimination compared with either sTfR or ferritin 


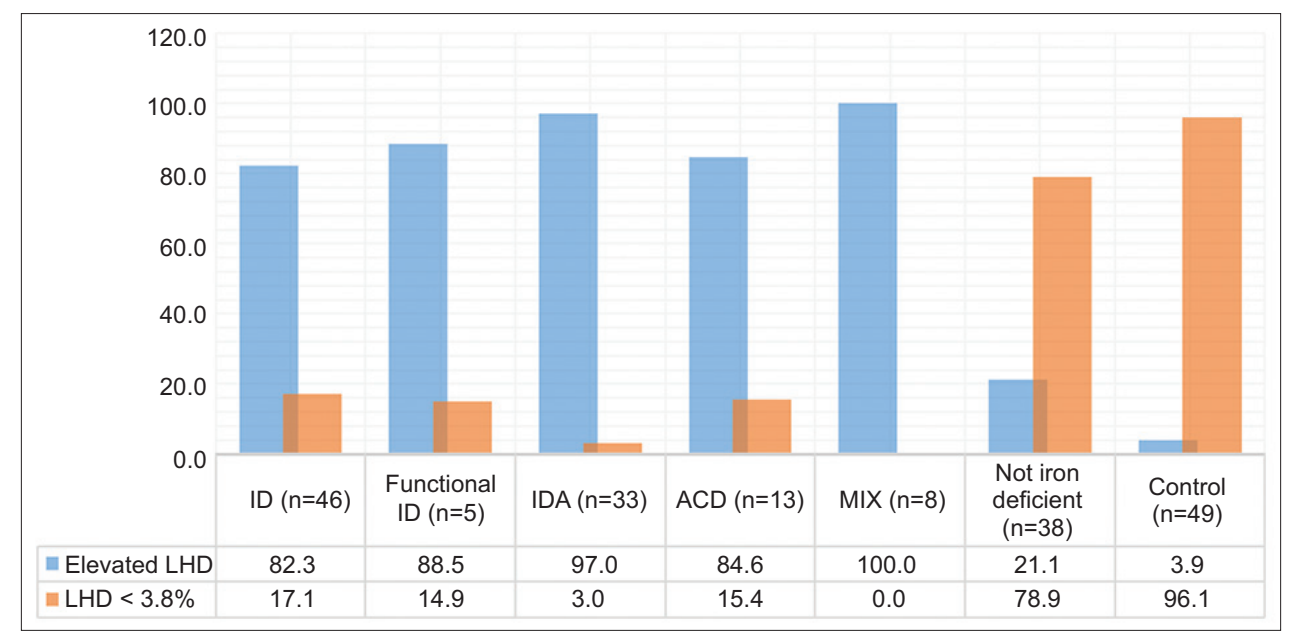

Figure 1 Percentage of elevated LHD\% in the study population

ID, iron deficiency; IDA, iron deficiency anemia; ACD, anemia of chronic disease; MIX, mixture of IDA and ACD; LHD, low hemoglobin density

alone, particularly in patients with chronic inflammatory disease [29-31]. However, TfR-F has limited availability and cost constraints [32]. Moreover, current assays are hampered by a lack of external quality controls and limited availability within the test repertoire of hospital-based laboratories [27,33,34].

Hypochromic red blood cells (\%HYPO), a measure of the percentage of erythrocytes with decreased hemoglobinization, has also been considered as a possible marker in this context. $\% \mathrm{HYPO}$ and reticulocyte indices perform very well in differentiating IDA and ACD [35,36]. However, patients with ACD may also have higher \%HYPO and lower reticulocytes than controls. This is due to iron-restricted erythropoiesis caused by the reduced availability of iron from body stores to red blood cells developing in the bone marrow. Furthermore, this approach is restricted by the fact that analyzers are available only from 2 manufacturers, Siemens and Sysmex.

The current gold standard in ID diagnosis, the determination of stainable iron strains in a bone marrow aspirate sample by Perls staining [37], is limited by factors such as incorrect aspiration of the bone marrow or insufficient sample size. Non-stainable iron stores seem to correlate well with true ID in patients with IDA. However, they can be misleading, since iron stores can even be stained in the bone marrow of patients with iron-restricted erythropoiesis, if adequately sampled. This reinforces current opinion among hematologists that a lack of detectable iron in bone marrow cannot be equated with ID, and that despite stringent criteria, bone marrow biopsy is not representative of a patient's true iron stores [38].

There is therefore a need to seek alternative iron status markers widely available. LHD\% was recently proposed as a potential indicator of iron available for erythropoiesis [16,17,39]. LHD $\%$ is a time-averaged measurement of the degree of hemoglobinization in mature erythrocytes. The analysis can be performed concurrently with routine blood counts at no additional cost and without additional blood collection [16]. $\mathrm{MCHC}$, the parameter from which LHD\% is calculated, is a stable laboratory parameter that can be reliably analyzed in

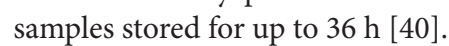

To our knowledge, this is the first study to highlight the value of LHD\% as an additional parameter to aid ID diagnosis in patients with IBD by evaluating iron status while incorporating the differentiation of absolute and functional ID. The results of our study confirmed that LHD\% is a reliable diagnostic marker of iron status in patients with IBD. LHD\% was found to be a more sensitive marker than hepcidin or bone marrow-stainable iron stores for identifying patients with iron-restricted erythropoiesis, especially if inflammation is present [18]. Our results indicate that LHD\% is suitable for the diagnosis of ID in patients with or without active inflammation. Furthermore, LHD\% was found to be superior to TSAT and s-ferritin in its diagnostic performance with regard to functional ID. Therefore, we propose that LHD\% may be a worthwhile additional diagnostic biomarker of ID in patients with IBD, especially when TSAT and s-ferritin are insufficiently accurate because of the presence of inflammation, offering additional diagnostic accuracy at no extra cost and without delay. Our ROC analysis identified 3.8\% as an optimal cutoff point for LHD\%, similar to the value of $4.0 \%$ suggested by Urrechaga et al $[16,17]$ for patients with chronic kidney disease. LHD\% has clinical utility, not only as a marker of iron-restricted erythropoiesis, but also in the discrimination between IDA and thalassemia, as shown by Urrechaga et al [16] and more recently by $\mathrm{Ng}$ et al [41].

As a general limitation, it must be kept in mind that, as a marker of cellular hypochromia, LHD\% is sensitive to the temperature and storage duration of the sample: LHD\% may be falsely elevated as a result of a reduction in $\mathrm{MCHC}$ associated with storage at room temperature or above. Therefore, if samples are kept at room temperature, LHD\% should be assessed within $6 \mathrm{~h}$ of collection. Refrigeration minimizes volume changes induced by sample storage, with hypochromia markers showing only minimal changes during the first $24 \mathrm{~h}$ after sample collection [42].

Our study has some limitations: Firstly, the study included a relatively small number of patients. Despite the relatively small number of samples, we determined a cutoff value close to that 
found in previous studies, and therefore see no restrictions in terms of reliability. Secondly, since LHD\% was not monitored after iron therapy, no statement can be made as to whether LHD\% may be a marker of therapeutic success. The lack of data on sTfR can be considered a further limitation; this was due to the fact that sTfR is not included in routine iron status assessments in the treatment center from which our patient data were obtained. Finally, no details are available concerning signs of clinical disease activity or disease duration at the time of blood sampling. Unfortunately, given the retrospective character of the study, it was not possible to collect these missing data.

In conclusion, in this study we demonstrated that LHD\% can verify the presence of ID, regardless of the presence of inflammation. LHD\% can be determined as part of routine laboratory tests at no extra cost and without additional blood collection, and thus offers a rapid, accurate and convenient additional diagnostic tool for ID in patients with IBD. We therefore propose that, using a cutoff value of $3.8 \%$, LHD\% holds promise as a simple, inexpensive and sensitive tool that allows an accurate and reliable diagnosis of ID with or without anemia in patients with inflammatory disorders.

\section{Summary Box}

\section{What is already known:}

- No reliable biochemical markers exist for the differentiation between iron deficiency anemia (IDA) and anemia of chronic disease in patients with inflammatory bowel disease (IBD)

- Low hemoglobin density (LHD\%) from Coulter counters has been suggested as a useful tool to detect iron deficiency, even in the context of inflammation

- Literature data suggest that LHD\% is more sensitive compared to ferritin, serum iron, transferrin saturation (TSAT), hepcidin or bone marrow iron stores in identifying patients with associated iron deficiency

\section{What the new findings are:}

- LHD\% had a better discriminating power than TSAT and s-ferritin in the diagnosis of IDA

- LHD\% was a reliable biomarker for the detection of iron deficiency in patients with IBD and anemia, regardless of whether inflammation is present

- LHD\% could provide added value in identifying patients with associated iron deficiency who could potentially benefit from parenteral iron replacement

\section{Acknowledgment}

The authors gratefully acknowledge the assistance of Janet Collins (Interdisziplinäres Crohn Colitis Centrum RheinMain, Frankfurt am Main, Germany) in correcting and proofreading the manuscript.

\section{References}

1. Koutroubakis IE, Ramos-Rivers C, Regueiro M, et al. Five-year period prevalence and characteristics of anemia in a large us inflammatory bowel disease cohort. J Clin Gastroenterol 2016;50:638-643.

2. Goodhand JR, Kamperidis N, Rao A, et al. Prevalence and management of anemia in children, adolescents, and adults with inflammatory bowel disease. Inflamm Bowel Dis 2012;18:513-519.

3. Filmann N, Rey J, Schneeweiss S, et al. Prevalence of anemia in inflammatory bowel diseases in European countries: a systematic review and individual patient data meta-analysis. Inflamm Bowel Dis 2014;20:936-945.

4. Bager P, Befrits R, Wikman O, et al. The prevalence of anemia and iron deficiency in IBD outpatients in Scandinavia. Scand J Gastroenterol 2011;46:304-309.

5. Aksan A, Wohlrath M, Iqbal TH, Farrag K, Dignass A, Stein J. Serum hepcidin levels predict intestinal iron absorption in patients with inflammatory bowel disease. Clin Lab 2019;65.

6. Aksan A, Wohlrath M, Iqbal TH, Dignass A, Stein J. Inflammation, but not the underlying disease or its location, predicts oral iron absorption capacity in patients with inflammatory bowel disease. J Crohns Colitis 2020;14:316-322.

7. Stein J, Aksan A, Farrag K, Dignass A, Radeke HH. Management of inflammatory bowel disease-related anemia and iron deficiency with specific reference to the role of intravenous iron in current practice. Expert Opin Pharmacother 2017;18:1721-1737.

8. Mast AE, Blinder MA, Lu Q, Flax S, Dietzen DJ. Clinical utility of the reticulocyte hemoglobin content in the diagnosis of iron deficiency. Blood 2002;99:1489-1491.

9. Coyne D. Iron indices: what do they really mean? Kidney Int Suppl 2006;(101):S4-S8.

10. Ganz T. Anemia of inflammation. N Engl J Med 2019;381:1148-1157.

11. World Health Organization. Serum ferritin concentrations for the assessment of iron status and iron deficiency in populations. 2011. Available from: https://www.who.int/vmnis/indicators/serum_ ferritin.pdf [Accessed 8 February 2021].

12. Cappellini MD, Comin-Colet J, de Francisco A, et al; IRON CORE Group. Iron deficiency across chronic inflammatory conditions: International expert opinion on definition, diagnosis, and management. Am J Hematol 2017;92:1068-1078.

13. Dignass A, Farrag K, Stein J. Limitations of serum ferritin in diagnosing iron deficiency in inflammatory conditions. Int $J$ Chronic Dis 2018;2018:9394060.

14. Peyrin-Biroulet L, Williet N, Cacoub P. Guidelines on the diagnosis and treatment of iron deficiency across indications: a systematic review. Am J Clin Nutr 2015;102:1585-1594.

15. Dignass AU, Gasche C, Bettenworth D, et al; European Crohn's and Colitis Organization [ECCO]. European consensus on the diagnosis and management of iron deficiency and anaemia in inflammatory bowel diseases. J Crohns Colitis 2015;9:211-222.

16. Urrechaga E. The new mature red cell parameter, low haemoglobin density of the Beckman-Coulter LH750: clinical utility in the diagnosis of iron deficiency. Int J Lab Hematol 2010;32(1 Pt 1):e144-e150. 
17. Urrechaga E, Unceta M, Borque L, Escanero JF. Low hemoglobin density potential marker of iron availability. Int J Lab Hematol 2012;34:47-51.

18. Martin-Cabrera P, Hung M, Ortmann E, et al. Clinical use of low haemoglobin density, transferrin saturation, bone marrow morphology, Perl's stain and other plasma markers in the identification of treatable anaemia presenting for cardiac surgery in a prospective cohort study. J Clin Pathol 2015;68:923-930.

19. Satsangi J, Silverberg MS, Vermeire S, Colombel JF. The Montreal classification of inflammatory bowel disease: controversies, consensus, and implications. Gut 2006;55:749-753.

20. WHO. Iron deficiency anaemia: assessment, prevention and control. Report of a joint WHO/UNICEF/UNU consultation. 1998. Available from: https:/www.who.int/nutrition/publications/ micronutrients/anaemia_iron_deficiency/WHO_NHD_01.3/en/ [Accessed 11 March 2021].

21. World Health Organization. Haemoglobin concentrations for the diagnosis of anaemia and assessment of severity. 2011. Available from: https://apps.who.int/iris/bitstream/handle/10665/85839/ WHO_NMH_NHD_MNM_11.1_eng.pdf?ua $=1$ [Accessed 8 February 2021].

22. Weiss G, Goodnough LT. Anemia of chronic disease. N Engl J Med 2005;352:1011-1023.

23. van Santen S, van Dongen-Lases EC, de Vegt F, et al. Hepcidin and hemoglobin content parameters in the diagnosis of iron deficiency in rheumatoid arthritis patients with anemia. Arthritis Rheum 2011;63:3672-3680.

24. Goodnough LT, Nemeth E, Ganz T. Detection, evaluation, and management of iron-restricted erythropoiesis. Blood 2010;116:4754-4761.

25. Bou-Fakhredin R, Halawi R, Roumi J, Taher A. Insights into the diagnosis and management of iron deficiency in inflammatory bowel disease. Expert Rev Hematol 2017;10:801-808.

26. Ott C, Liebold A, Takses A, Strauch UG, Obermeier F. High prevalence but insufficient treatment of iron-deficiency anemia in patients with inflammatory bowel disease: results of a populationbased cohort. Gastroenterol Res Pract 2012;2012:595970.

27. Thomas DW, Hinchliffe RF, Briggs C, et al. Guideline for the laboratory diagnosis of functional iron deficiency. $\mathrm{Br} J$ Haematol 2013;161:639-648.

28. Feelders RA, Vreugdenhil G, Eggermont AM, Kuiper-Kramer PA, van Eijk HG, Swaak AJ. Regulation of iron metabolism in the acute-phase response: interferon gamma and tumour necrosis factor alpha induce hypoferraemia, ferritin production and a decrease in circulating transferrin receptors in cancer patients. Eur J Clin Investig 1998;28:520-527.
29. Suominen P, Möttönen T, Rajamäki A, Irjala K. Single values of serum transferrin receptor and transferrin receptor ferritin index can be used to detect true and functional iron deficiency in rheumatoid arthritis patients with anemia. Arthritis Rheum 2000;43:1016-1020.

30. Punnonen K, Irjala K, Rajamäki A. Serum transferrin receptor and its ratio to serum ferritin in the diagnosis of iron deficiency. Blood 1997;89:1052-1057.

31. Rimon E, Levy S, Sapir A, et al. Diagnosis of iron deficiency anemia in the elderly by transferrin receptor-ferritin index. Arch Intern Med 2002;162:445-449.

32. Abitbol V, Borderie D, Polin V, et al. Diagnosis of iron deficiency in inflammatory bowel disease by transferrin receptor-ferritin index. Medicine (Baltimore) 2015;94:e1011.

33. Koulaouzidis A, Cottier R, Bhat S, Said E, Linaker BD, Saeed AA. A ferritin level $>50$ microg/L is frequently consistent with iron deficiency. Eur J Intern Med 2009;20:168-170.

34. Koulaouzidis A, Saeed AA, Abdallah M, Said EM. Transferrin receptor level as surrogate peripheral blood marker of iron deficiency states. Scand J Gastroenterol 2009;44:126-127.

35. Syed S, Kugathasan S, Kumar A, et al. Use of reticulocyte hemoglobin content in the assessment of iron deficiency in children with inflammatory bowel disease. J Pediatr Gastroenterol Nutr 2017;64:713-720.

36. Urrechaga E, de la Hera P, Aguayo FJ. Reticulocyte hemoglobin and hypochromic erythrocytes in the study of erythropoiesis in patients with inflammatory bowel disease. Scand J Clin Lab Invest 2019;80:124-128.

37. Hughes DA, Stuart-Smith SE, Bain BJ. How should stainable iron in bone marrow films be assessed? J Clin Pathol 2004;57:1038-1040.

38. Barron BA, Hoyer JD, Tefferi A. A bone marrow report of absent stainable iron is not diagnostic of iron deficiency. Ann Hematol 2001;80:166-169.

39. Urrechaga E, Borque L, Escanero JF. Percentage of hypochromic erythrocytes as a potential marker of iron availability. Clin Chem Lab Med 2012;50:685-687.

40. Jackson C, Best N, Elliott P. UK Biobank Pilot Study: stability of haematological and clinical chemistry analytes. Int J Epidemiol 2008;37 Suppl 1:i16-i22.

41. Ng EH, Leung JH, Lau YS, Ma ES. Evaluation of the new red cell parameters on Beckman Coulter DxH800 in distinguishing iron deficiency anaemia from thalassaemia trait. Int $J$ Lab Hematol 2015;37:199-207.

42. Archer NM, Brugnara C. Diagnosis of iron-deficient states. Crit Rev Clin Lab Sci 2015;52:256-272. 